\title{
Verändern statt verhindern
}

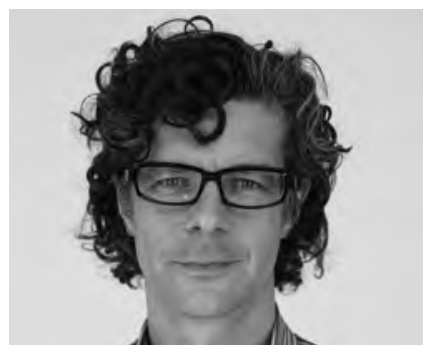

VON CARSTEN TESCH

Carsten Tesch ist Journalist und Hörfunk-Moderator bei »Figaro", dem Kulturradio des Mitteldeutschen Rundfunks. Daneben arbeitet er als Berater für Medienarbeit und Organisationsentwicklung in sozialen Organisationen sowie als Coach in PR-Krisen und Konfliktsituationen.

E-Mail Info@Tesch-Texter.de

\author{
Die »Instrumentenreform» in der Beschäftigungs- \\ förderung ist beschlossene Sache. Die gemeinnützigen \\ Verbände und Träger in der Sozialwirtschaft sollten \\ untersuchen, warum die Proteste dagegen gescheitert \\ sind und dann daraus die richtigen Schlüsse ziehen.
}

Die Bundesregierung kürzt Milliarden bei der Arbeitsmarktpolitik. Die Wohlfahrtsverbände reagieren alarmiert. Der Protest ist breit und namhaft. Eine Springflut von vernichtenden Stellungnahmen läuft auf Berlin zu. Es dürfte keinen Bundestagsabgeordneten gegeben haben, der keine Post zur Instrumentenreform bekommen hat. Mitten in der Ferienzeit unterschreiben über 3.000 Fachleute kurzfristig den Appell von Paritätischem Wohlfahrtsverband und Deutschen Gewerkschaftsbund. Aber die Öffentlichkeit bleibt gelassen, denn in den Zeitungen steht wenig dazu, der Tagespresse entgeht die Dimension der Reform.

\section{Erste These: Die Arbeitslosen- zahlen waren zu gut}

Was ist da passiert? Fragt man bei den Verbänden, dann lag es an den guten Arbeitsmarktzahlen, die Brisanz gekostet hätten. An der Nachrichtenlage mit der Finanzkrise und dann auch noch mit dem Papst im Bundestag am Tag der Abstimmung. Und überhaupt sei das alles sehr komplex und Journalisten schwer zu vermitteln.

Fragte man Journalisten in Berlin, konnte es passieren, dass sie noch gar nicht davon gehört hatten. Andere sahen in dem Konflikt über die Instrumentenreform einfach einen Verteilungskampf. Ein Ritual zwischen Kostenträger und Leistungserbringer, wo beide Seiten gute Gründe haben. Nicht mehr als ein Knirschen im System, was regelmäßig vorkommt, da die Mittel eben begrenzt sind.
Interessant auch, dass offenbar niemand auffiel, wie sehr der Gesetzentwurf die Träger diskreditiert. Ressentiments gegen den dritten Sektor werden darin als Tatsachen behandelt. Daraus wird eine Wirklichkeit konstruiert, mit Mitnahme- und Klebeeffekten, Wettbewerbsverzerrung und sinnlosen Maßnahmen (mit Plastikobst und Puzzlespielen), die das neue Gesetz verbessern soll. Als wären mangelnde Transparenz, Verschwendung und Missbrauch die Regel.

\section{Zweite These: Über Geld lässt sich schlecht streiten}

Und schließlich hat auch der Protest selbst perfekt in dieses schiefe Bild gepasst. Denn was hat die Wohlfahrt auf die Barrikaden gebracht? Es ist schwer zu übersehen, dass der Sturm der Entrüstung genau in dem Moment losbrach, als das Arbeits- und Sozialministerium andeutete, am großen Hahn für die arbeitsmarktpolitischen Maßnahmen drehen zu wollen. Der Verdacht lag nahe, dass die »Gemeinnützigen « da vor allem ihre eigenen Felle wegschwimmen sahen. Dass sie um ihr Stück vom großen Kuchen fürchteten. Dass sie vor allem ihre eigene weit verzweigte Trägerlandschaft verteidigten mit Umsätzen, Arbeitsplätzen, Einfluss.

Mal wieder schien es, »nur ums Geld« zu gehen. Das ist das Problem. Über Geld lässt sich einfach schlecht streiten. Öffentlich, vor Publikum, in den Arenen der medialen Meinungsbildung. Ist Geld im Spiel, ist keiner mehr ehrlich. Unsere Sehgewohnheiten rechnen unwillkürlich Befangenheiten 
ein. Die Konsequenzen der öffentlichen Verteilungskämpfe können gravierend sein, wo es »nur ums Geld zu gehen scheint «, wird sich die Anteilnahme des Publikums in Grenzen halten.

\section{Dritte These: Öffentlichkeitsarbeit wird vernachlässigt}

Die Wohlfahrtsverbände aber erleben wir dauernd in Mitteldiskussionen. Das wäre ein spannendes Forschungsthema, zu untersuchen, wie viele ihrer öffentlichen Äußerungen sich um Budgets und Kürzungen drehen. Polemisch verkürzt gesagt: Man hört und sieht sie wenig, aber geht's ans Geld, gibt's plötzlich Alarm. Plätschert die Öffentlichkeitsarbeit sonst so vor sich hin, hat sie auf einmal Priorität. Einrichtungen und Verbände entdecken ihre leidenschaftliche Seite und machen mobil. Wie bei den Protesten gegen die Instrumentenreform.

Die Öffentlichkeit aber bleibt skeptisch. Sie nimmt der Wohlfahrt die Gemeinnützigkeit, das öffentliche Interesse, die anwaltschaftliche Vertretung im Namen der Betroffenen nicht so ohne weiteres ab. Es fällt schwer, Soziale Arbeiter und Arbeitslose im selben Boot zu sehen. Politik und Verbände scheinen da viel besser zusammenzupassen.

\section{Vierte These: Die Politiknähe kostet Vertrauen}

Man muss sich die Formen des Protestes anschauen: Die Papierflut, die die Verbände produziert haben, war fast ausschließlich an die Politik adressiert. An Gremien, Ausschüsse, Hintergrundrunden. Außerhalb des Systems Kostenträger/Leistungsträger hat das niemand verstanden. Dem breiten Publikum gegenüber tat sich der Protest schwer, eine Sprache zu finden. Kurz: unbeholfen in der Öffentlichkeit, aber gremienaffin und agil in der Lobbyarbeit. Das passt zu Konzernen und Industrieverbänden, die Gemeinnützigen kostet diese Nähe zur Politik Glaubwürdigkeit.

Meiner Ansicht nach aber ist entscheidend, dass sich die Wohlfahrt genau in dem Moment zu rühren begann, wo die Arbeitsmarktpolitik der Bundesregierung wieder konkrete Sparpolitik geworden ist. Als es ums Geld ging. Hätte es nicht auch ohne die Instrumentenreform dringend Anlass gegeben, das Thema Langzeitarbeitslosigkeit vehement zu verfolgen?

Die Träger in der Arbeitsmarktpolitik wissen schon lange, dass Vollbeschäftigung eine Illusion ist. Dass es hunderttausende Menschen gibt, die auf dem ersten Arbeitsmarkt niemals zu vermitteln sind. Dass viele von ihnen aber eine Form von Beschäftigung brauchen, weil wir nun mal in einer Arbeitsgesellschaft leben und es uns schwerfällt, uns anders als nützlich zu verstehen.

Die Träger wissen auch, wie Arbeitslose von den Agenturen behandelt werden. Wie viel Willkür es dort gibt. Dass für arbeitslose Menschen die Bürgerrechte nur noch beschränkt gelten. Den Trägern könnte auch auffallen, dass Arbeitslose nach wie vor öffentlich verunglimpft werden. Wo sonst Political Correctness durchgesetzt ist, dürfen sich Arbeitslose immer noch anhören, dass sich Leistung wieder lohnen muss. Wer von den Verbänden arbeitet wirklich daran, diese »Nur-zu-faul-zum-Arbeiten «Klischees infrage zu stellen?

\section{Fünft These: Wir brauchen eine anwaltschaftliche Agenda}

Wie auch immer, die »Reform « kommt. Welche Schlüsse sollte die Wohlfahrt aus den gescheiterten Protesten ziehen? Sie kann sich jetzt nicht mit dem Gesetz einrichten und das öffentliche Engagement für Langzeitarbeitslose erst mal wieder vertagen. Dann wäre es wirklich nur um Besitzstandwahrung gegangen. Das Beispiel zeigt, dass sich die Sozialen vielmehr von der »Agenda der Grausamkeiten ", die von der Politik bestimmt wird, abgrenzen sollten. Dass sie alternativ eine eigene Tagesordnung relevanter Themen entwickeln sollten. Von Verhindern umstellen auf Verändern.

Für die Arbeitsmarktpolitik hieße das, jetzt, wo es finanziell erst mal nichts mehr zu gewinnen gibt, trotzdem an dem Thema Langzeitarbeitslosigkeit dranzubleiben. Die Nachricht, dass Ursula von der Leyen mindestens 400.000 Langzeitarbeitslose als nicht mehr systemrelevant praktisch abgemeldet hat, ist ja in der Öffentlichkeit noch gar nicht angekommen. Das wäre also dringend nachzuholen. Die Bundesregierung hat sich von der Illusion der Vollbeschäftigung praktisch verabschiedet. Das sollte die Gesellschaft erfahren.

Außerdem haben die Träger mit dem
»Passiv-aktiv-Modell (PAT) « eine konkrete Alternative anzubieten. Die Passivleistungen aus dem Hartz IV-System wären danach in Aktivleistungen umzuwidmen, in Lohn, mit dem öffentliche Beschäftigung ohne zusätzliche Kosten bezahlt werden könnte. Eine Rechnung, die auf einen Bierdeckel passt. Eine Position, die unmittelbar plausibel wirkt. Die Bundesregierung hat keine überzeugenden Argumente dagegen, denn öffentlich geförderte Beschäftigung ist ein Tabu in der Koalition. So ließe sich anhand des PAT-Modells gut vermitteln, mit welchen neoliberalen Vorurteilen, es die Gemeinnützigen zu tun haben. Aus welcher ideologischen Ecke das Misstrauen und die Herablassung gegen den dritten Sektor eigentlich kommt.

Und schließlich ist dieses Gesetz eine ideale Vorlage, danach zu fragen, was das über die Arbeitsgesellschaft sagt, wenn so viele Mitbürger gar keine Chance auf Teilhabe haben. Was es über das mentale Muster von Arbeit und Leistung sagt. Ist es nicht höchste Zeit, dieses Muster, wonach sich der Wert eines Menschen letztlich nach seiner Arbeitskraft bemisst, offensiv infrage stellen?

\section{Resümee}

$\mathrm{Zu}$ erkennen, dass sich über Geld schlecht streiten lässt, hieße also für die Wohlfahrt der Öffentlichkeitsarbeit ständig Priorität einzuräumen und nicht nur in Verteilungskrisen. Würde bedeuten, viel unbeirrter als bisher, eine eigene anwaltschaftliche Agenda zu verfolgen. Sich außerdem stärker als Nichtregierungsorganisationen abzugrenzen gegen die staatliche Hand, die einen füttert. Und schließlich selbstbewusst den kapitalismusskeptischen Zeitgeist zu nutzen und den dritten Sektor als gleichwertige Alternative zur freien Wirtschaft zu profilieren. Statt ums Geld zu streiten, immer unter Verdacht, dass es eigentlich nur darum geht, würde eine Agenda in den Vordergrund treten, mit der sich vielleicht Gesellschaft verändern, bestimmt aber Glaubwürdigkeit gewinnen ließe. 